\title{
Low shear stress induces endothelial cell apoptosis and monocyte adhesion by upregulating PECAM-1 expression
}

\author{
XIANGRONG XIE $^{1,2^{*}}$, FENG WANG $^{1 *}$, LINLIN ZHU $^{1}$, HONGFENG YANG $^{1}$, DAORONG PAN $^{1}$, \\ YAN LIU ${ }^{1}$, XINLIANG QU ${ }^{1}$, YUE GU ${ }^{1}, \mathrm{XIAOBO} \mathrm{LI}^{1}$ and SHAOLIANG CHEN ${ }^{1}$ \\ ${ }^{1}$ Department of Cardiology, Nanjing First Hospital, Nanjing Medical University, Nanjing, Jiangsu 210006; \\ ${ }^{2}$ Department of Cardiology, The First Affiliated Hospital, Yijishan Hospital of Wannan Medical College, \\ Wuhu, Anhui 241001, P.R. China
}

Received October 13, 2019; Accepted March 19, 2020

DOI: $10.3892 / \mathrm{mmr} .2020 .11060$

\begin{abstract}
Low shear stress serves an important role in the initiation and progression of atherosclerotic lesions, with an impact on progression, but its detailed mechanisms are .not yet fully known. The present study aimed to investigate endothelial cell (EC) apoptosis, as well as monocyte adhesion induced by low shear stress and the potential underlying mechanisms. The expression of platelet endothelial cell adhesion molecule-1 (PECAM-1) was demonstrated to be enhanced in human umbilical vascular ECs with a trend that was associated with time when stimulated by low shear stress compared with unstimulated cells. EC apoptosis was increased under low shear stress compared with unstimulated cells, and knockdown of PECAM-1 inhibited this process. Furthermore, downregulation of PECAM-1 reduced monocyte adhesion induced by low shear stress compared with that in the negative control cells. Mechanistically, PECAM-1 small interfering RNA transfection increased Akt and forkhead box O1 phosphorylation under low shear stress conditions compared with that in the negative control cells. Collectively, the findings of the present study revealed that low shear stress induced EC apoptosis and monocyte adhesion by upregulating PECAM-1 expression, which suggested that PECAM-1 may be a potential therapeutic target for atherosclerosis.
\end{abstract}

\section{Introduction}

As a leading cause of cardiovascular disease, atherosclerosis is a serious health hazard in humans (1). Endothelial dysfunction,

Correspondence to: $\mathrm{Dr}$ Shaoliang Chen, Department of Cardiology, Nanjing First Hospital, Nanjing Medical University, 68 Changle Road, Nanjing, Jiangsu 210006, P.R. China

E-mail: chmengx@126.com

*Contributed equally

Key words: platelet endothelial cell adhesion molecule-1, low shear stress, atherosclerosis, endothelial cell apoptosis, monocyte adhesion characterized by changes in sensitivity to apoptosis, coagulation, inflammatory activity, barrier function and proliferation, is the first step in atherosclerosis development $(2,3)$. Recent advances made regarding atherosclerosis have emphasized that improvement of endothelial function is a potential target for atherosclerosis treatment (4).

Increasing evidence has demonstrated that the endothelial dysfunction process is directly influenced by various factors, including nitric oxide dysfunction, epigenetic factors, oxidative stress, inflammatory factors and low shear stress (LSS) (5-11). Among these, LSS, tangential stress produced by endothelial surface blood flow friction, which is observed at bifurcations, branching points and inside areas of curved segments of the coronary artery, induces inflammation and apoptosis, and subsequently disrupts the endothelial barrier, eventually leading to atherosclerosis (11-15). However, to date, no molecular targets have clearly linked LSS with the endothelial barrier. Thus, it is important to identify potential molecular targets for the improvement of atherosclerosis treatment.

As a molecule that causes cell adhesion, the expression of platelet endothelial cell adhesion molecule-1 (PECAM-1, also termed CD31) is ubiquitous in various types of cells, including monocytes, neutrophils, $\mathrm{T}$ cells and endothelial cells (ECs), and is crucial for vascular barrier function regulation as a result of various stimuli, including shear stress (16). Under certain physiological conditions, the endothelial barrier function is supported by PECAM-1 by controlling the junctional and adhesive properties of ECs (17). During inflammation observed in vessels affected by atherosclerosis, the function of PECAM-1 is impaired, leading to an increase in neutrophils and other leukocy tes adhering to ECs, vascular integrity loss and increased transmigration of leukocytes to the intima media (18). Of note, increasing evidence from in vivo experiments has indicated that PECAM-1 removal leads to a decrease in the development of plaque in LSS environments (19-21). These observations indicate a proatherogenic role of PECAM-1 under LSS, but its mechanism of action has not yet been elucidated. The present study hypothesized that LSS may upregulate PECAM-1 expression, leading to EC apoptosis and monocyte adhesion that subsequently disrupts the endothelial barrier, resulting in atherosclerosis. 


\section{Materials and methods}

Reagents. Antibodies against PECAM-1 (cat. no. 77699S), PECAM-1 (cat.no.3528S), phospho-Akt (Ser473; cat. no.4060S), phospho-Akt (Thr308; cat. no. 13038S), Akt (cat. no. 9272S), phospho-forkhead box O (FoxO1; Ser256; cat. no. 84192S), FoxO1 (cat. no. 2880S) and $\beta$-actin (cat. no. 8457S) were purchased from Cell Signaling Technology, Inc. FITC-labeled goat anti-rabbit IgG (cat. no. GB22303) and horseradish peroxidase (HRP)-labeled goat anti-rabbit IgG (cat. no. GB23303) were purchased from Wuhan Servicebio Technology Co., Ltd. FBS, DMEM and RPMI-1640 media were purchased from Gibco; Thermo Fisher Scientific, Inc. The Annexin V-FITC/propidium iodide (PI) Apoptosis Detection kit was purchased from BD Bioscience.

Cell culture. The EA.hy926 cells, obtained from The Type Cell Bank of Culture Collection of the Chinese Academy of Sciences, are a human umbilical vein EC (HUVEC) line. The cells were cultured in 10\% FBS-supplemented DMEM in a 5\% $\mathrm{CO}_{2}$ incubator at $37^{\circ} \mathrm{C}$. The American Type Culture Collection was the source of the human monocytic leukemia cell line THP-1. RPMI-1640 medium supplemented with 10\% FBS was used to culture the THP- 1 cells in a $5 \% \mathrm{CO}_{2}$ incubator at $37^{\circ} \mathrm{C}$.

Small interfering RNA (siRNA) transfection. The siRNA sequences used in the present study were as follows: PECAM-1 forward, 5'-AUUCUGGUCUCGAGAAUUCUU-3' and reverse, 5'-GAAUUCUCGAGACCAGAAUUU-3'; and PECAM-1 negative control (NC) forward, 5'-ACGUGA CACGUUCGGAGAATT-3' and reverse, 5'-UUCUCCGAA CGUGUCACGUTT-3' (Shanghai GenePharma Co., Ltd.). The Lipofectamine ${ }^{\circledR}$-RNAiMAX transfection reagent (Invitrogen; Thermo Fisher Scientific, Inc) was utilized to transfect the HUVECs with PECAM-1 or NC siRNA as previously described (15). Cells at 70-80\% confluency were co-cultured with $50 \mathrm{nmol} / 1 \mathrm{PECAM}-1$ or $50 \mathrm{nmol} / 1 \mathrm{NC}$ siRNA and Lipofectamine ${ }^{\circledR}$-RNAiMAX at $37^{\circ} \mathrm{C}$ for $6 \mathrm{~h}$. At $48 \mathrm{~h}$ post-transfection, the cells were either exposed to shear stress or not. The experiments were performed in triplicate.

Shear stress experiment. Shear stress of $2 \mathrm{dyn} / \mathrm{cm}^{2}$ was applied in vitro using a parallel-flow chamber as previously described $(15,22,23)$. In brief, endothelial monolayers containing cell culture slides were subjected to LSS for 0 , 30,60 and 120 min after being placed in a parallel-plate flow chamber. Prior to the flow experiments, the cells were transferred to serum-free DMEM for 120 min to maintain their quiescence.

Western blotting analysis. Total protein of the HUVECs was obtained as previously described (15). The protein expression of PECAM-1, FoxO1, phospho-FoxO1, Akt, and phospho-Akt in HUVECs were analyzed using western blotting. SDS-PAGE (10\% gel) was used to separate an equal quantity $(40 \mu \mathrm{g})$ of proteins, which were then transferred onto PVDF membranes. After blocking with 5\% BSA (cat. no. SBJ-DB2040; Nanjing SenBeiJia Biological Technology Co., Ltd.) for $2 \mathrm{~h}$ at $25^{\circ} \mathrm{C}$, incubation of the membranes was performed at $4^{\circ} \mathrm{C}$ with anti-PECAM-1 $(1: 1,000)$, anti-FoxO1
(1:1,000), anti-phospho-FoxO1 (1:1,000), anti-Akt $(1: 1,000)$, anti-phospho-Akt Ser473 (1:1,000), anti-phospho-Akt Thr308 $(1: 1,000)$ and anti- $\beta$-actin $(1: 1,000)$ antibodies overnight. The membranes were washed with TBS with $0.1 \%$ Tween-20 (cat. no. T8220; Beijing Solarbio Science \& Technology Co., Ltd.) and incubated with HRP-conjugated secondary antibodies $(1: 10,000)$ for $2 \mathrm{~h}$ at $25^{\circ} \mathrm{C}$. The antibody-bound target antigens were detected using enhanced chemiluminescence reagents (cat. no. WBKLS0500; EMD Millipore). A GeneGnome chemiluminescence imaging system (Syngene) was used to capture images, and ImageJ software (v.1.8.0; National Institutes of Health) was used to analyze the target protein expression. The experiments were performed at least in triplicate.

Adhesion assay. The effects of LSS on THP-1 cell adherence onto HUVEC monolayers was assessed by an adhesion assay. Following exposure of the HUVECs to LSS for $60 \mathrm{~min}$, the HUVECs were covered with THP-1 cells $(10,000$ cells $/ \mathrm{ml})$ and co-incubated for $1 \mathrm{~h}$ at $37^{\circ} \mathrm{C}$. Attached THP-1 cell quantity was detected using an IX73P2F light microscope (Olympus Corporation), magnification $\mathrm{x} 200$. The adherent cell quantity was determined in three high-power microscopic fields that were randomly chosen.

Flow cytometry for apoptosis analysis. HUVEC early and late apoptosis determination was conducted using an Annexin V-FITC/PI Apoptosis Detection kit according to the manufacturer's instructions. In brief, LSS was used to stimulate the HUVECs for $90 \mathrm{~min}$, and subsequently Annexin V-FITC/PI was used to stain the cells at $37^{\circ} \mathrm{C}$ for $30 \mathrm{~min}$. The HUVECs were washed twice with PBS, and cold PBS was used to resuspend the stained cells. The BD FACSCanto II system (BD Biosciences) was used to perform the flow cytometry, and BD FACSDiva Software (version 8.0.1; BD Biosciences) was used to analyze the data.

Immunofluorescence. The HUVECs were fixed in $4 \%$ paraformaldehyde for $20 \mathrm{~min}$ at $25^{\circ} \mathrm{C}$, permeabilized with $0.1 \%$ Triton X-100/PBS for $10 \mathrm{~min}$ and blocked with 5\% BSA for $30 \mathrm{~min}$ at $25^{\circ} \mathrm{C}$. Subsequently, the HUVECs were incubated with a primary rabbit anti-FoxO1 monoclonal antibody (1:100) overnight at $4^{\circ} \mathrm{C}$. After three washes with PBS and incubation with FITC-labeled goat anti-rabbit $\operatorname{IgG}(1: 200)$ secondary antibody for $120 \mathrm{~min}$ at $25^{\circ} \mathrm{C}$, DAPI (1:1; Beyotime Institute of Biotechnology) was used to incubate the slides according to the manufacturer's instructions. Images were captured using an LSM 880 (Carl Zeiss AG) laser scanning confocal microscope (magnification, x630; 3 fields analyzed per sample).

Animals and surgical procedures. The Institutional Animal Care and Use Committee of Nanjing Medical University approved the in vivo experimental procedure (approval no. SYXK2016-0006). According to previous studies $(15,24), 8$ week-old male C57BL/6 mice $(n=8$; Nanjing Medical University Animal Center, Nanjing, China), weighing $\sim 25 \mathrm{~g}$, fed with a standard chow diet and adequate water, housed in specific pathogen-free facilities with a $12 \mathrm{~h}$ light/dark cycle, $25-26^{\circ} \mathrm{C}$ controlled temperature, standard atmospheric pressure and $50 \%$ humidity, were euthanized 
A

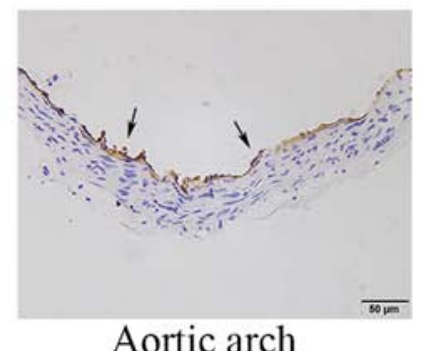

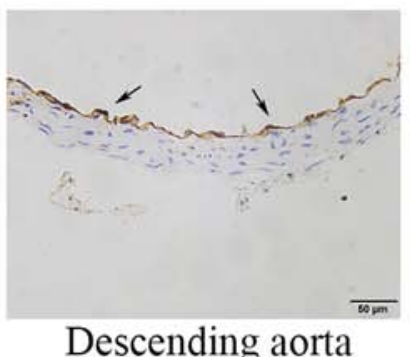

Descending aorta

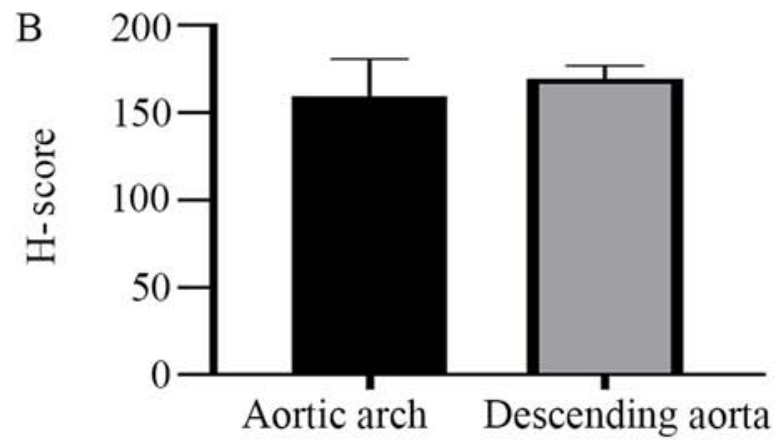

Figure 1. PECAM-1 expression in mouse aortic ECs. (A) Immunohistochemical staining of PECAM-1 expression (arrowheads) in luminal ECs of the descending aorta and the aortic arch in wild-type mice. Original magnification, $\mathrm{x} 400$; scale bar, $50 \mu \mathrm{m}$. (B) Semi-quantification of PECAM-1 expression using the H-Score. PECAM-1, platelet endothelial cell adhesion molecule-1; EC, endothelial cells; H-score, hormone score.

with an intraperitoneal injection of $200 \mathrm{mg} / \mathrm{kg}$ sodium pentobarbital. Cold PBS was used to rinse the aortas three times by inserting a cannula into the left ventricle. The aortic arch and the descending aorta were separated and fixed in $4 \%$ paraformaldehyde for $24 \mathrm{~h}$ at $25^{\circ} \mathrm{C}$, and then embedded in paraffin.

Immunohistochemistry of tissues. Fixed and paraffinembedded aortic tissues were sectioned at $5 \mu \mathrm{m}$. Following previously described protocols (25), immunochemistry analysis was conducted. PBS was used to rinse the aortic slices, followed by $30 \mathrm{~min}$ of $3 \% \mathrm{H}_{2} \mathrm{O}_{2}$ treatment at $25^{\circ} \mathrm{C}$ for quenching endogenous peroxidase activity and subsequent incubation for $1 \mathrm{~h}$ at $25^{\circ} \mathrm{C}$ with $5 \%$ BSA supplemented with $0.3 \%$ Triton $\mathrm{X}-100$. The sections were incubated overnight at $4^{\circ} \mathrm{C}$ using a rabbit anti-PECAM-1 antibody (1:100) for the detection of PECAM-1. PBS was used for control staining. Incubation with HRP-labeled goat anti-rabbit IgG (1:200) was conducted for $1 \mathrm{~h}$ at $25^{\circ} \mathrm{C}$ for visualization. Images were captured using a BX43 light microscope (Olympus Corporation) at magnification $\mathrm{x} 400$, and CellSens Standard software (version 1.14; Olympus Corporation). The hormone receptor score (H-Score) was used to evaluate PECAM-1 expression, as previously described (26).

Statistical analysis. Data are presented as the mean \pm SD of each experiment performed in triplicate. ANOVA followed by post hoc Turkey's test or Student's t-test were performed using GraphPad Prism (version 8.0.1; GraphPad Software, Inc.). $\mathrm{P}<0.05$ was considered to indicate a statistically significant difference.

\section{Results}

LSS enhances PECAM-1 expression in ECs. In order to observe the differences in PECAM-1 expression between the descending aorta and the aortic arch, the entire mouse aortic arch from the aortic valve to the descending thoracic aorta was serially sectioned, and the cross-sections were stained by immunohistochemistry. PECAM-1 was expressed in aortic ECs of mice and was observed at similar levels in the descending aorta and the aortic arch in the cross-sectional analysis (Fig. 1). To further investigate the effects of LSS on PECAM-1 expression, in vitro flow experiments were performed using a parallel-plate flow chamber system. LSS increased the PECAM-1 protein level in the HUVECs in a time-dependent manner (Fig. 2A and B), and this effect was attenuated by transfection with PECAM-1 siRNA (Fig. 2C and D).

PECAM-1 knockdown suppresses LSS-induced adhesion of monocytes to HUVECs. Under LSS conditions, monocytes easily adhere to ECs (27). Atherosclerosis initiation involves the adhesion of monocytes to the endothelium, which can be induced by LSS (27-29). Therefore, the present study examined whether PECAM-1 regulated monocyte adhesion to HUVECs. THP-1 cell adhesion to the HUVECs was significantly increased by LSS compared with the unstimulated HUVECs, and this effect was inhibited by PECAM-1 siRNA transfection (Fig. 3). These results suggested that PECAM-1 promoted LSS-induced adhesion of monocytes to HUVECs.

PECAM-1 knockdown inhibits LSS-induced apoptosis of HUVECs. LSS is an identified risk factor for atherosclerosis, which results in the apoptosis of ECs (30). Flow cytometry was used to analyze the effects of PECAM-1 impact on HUVEC apoptosis induced by LSS. LSS induced a significant increase in HUVEC apoptosis compared with unstimulated cells (Fig. 4A and B). PECAM-1 siRNA transfection decreased the LSS-induced apoptosis of the HUVECs compared with the NC siRNA-transfected cells (Fig. 4A and B). These results suggested that PECAM-1 increased the LSS-induced apoptosis of HUVECs.

PECAM-1 knockdown increases LSS-induced Akt and FoxO1 phosphorylation in HUVECs. LSS induced Akt phosphorylation at Ser473 and Thr308 and FoxO1 phosphorylation at Ser256. PECAM-1 siRNA transfection enhanced the effects of LSS-induced phosphorylation of Akt and FoxO1 in HUVECs compared with NC siRNA-transfected cells (Fig. 5A-D). These results indicated that both Akt and FoxO1 were regulated by PECAM-1 under LSS conditions. In order to further define the nuclear or cytoplasmic relocation of FoxO1 under LSS conditions with or without PECAM-1 siRNA transfection, immunofluorescence staining was performed. LSS induced nucleocytoplasmic transportation of FoxO1 compared with untreated cells, and this effect was enhanced by PECAM-1 siRNA transfection (Fig. 5E). These results suggested that PECAM-1 knockdown inhibited LSS-induced apoptosis of HUVECs by enhancing Akt and FoxO1 phosphorylation. 
A
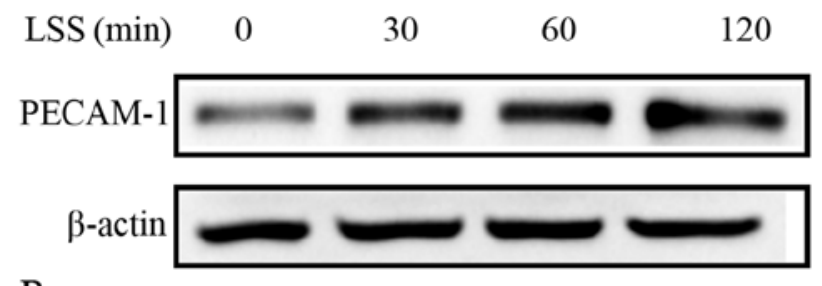

B

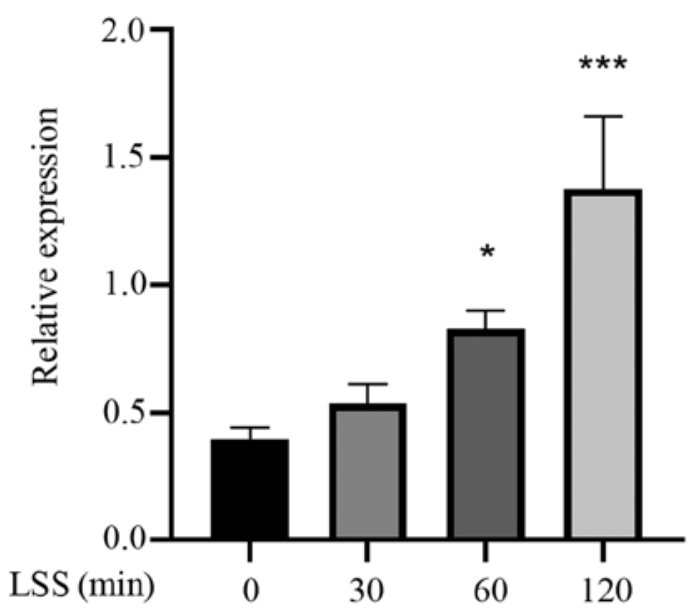

$\mathrm{C}$
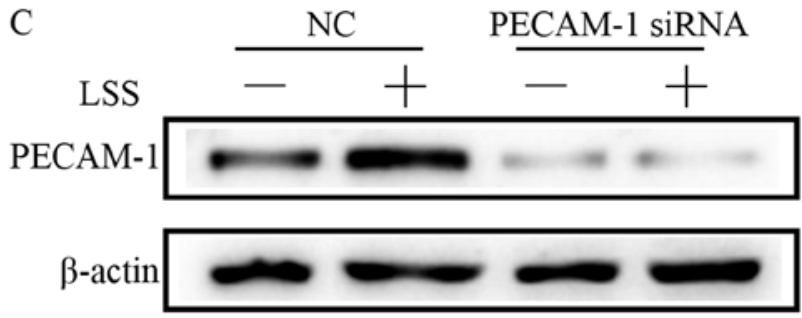

D

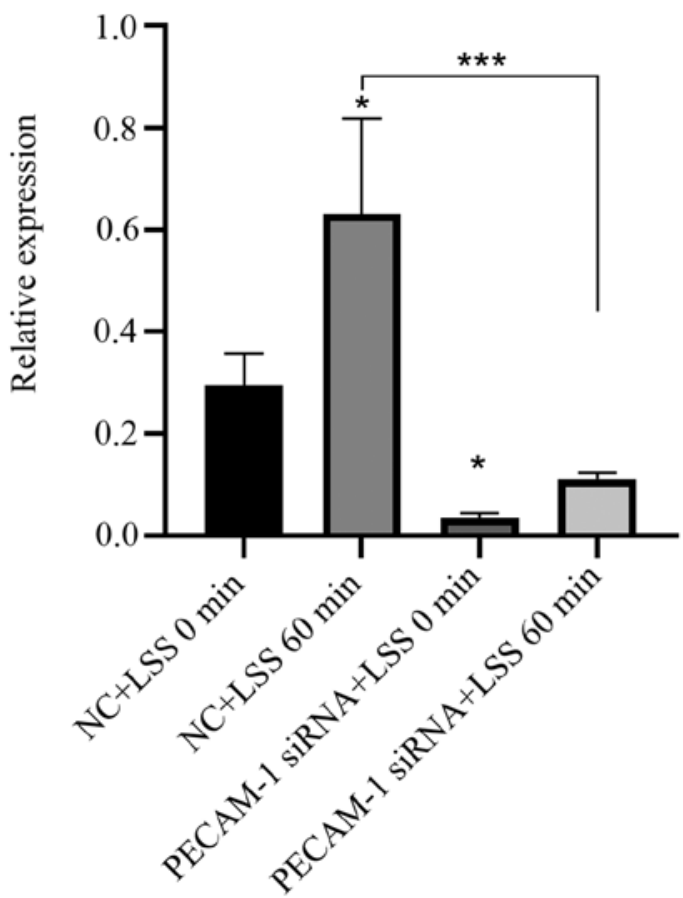

Figure 2. LSS increases PECAM-1 expression in HUVECs. (A) Representative immunoblots and (B) quantitative analysis of PECAM-1 after 0, 30, 60, and 120 min of HUVEC exposure to LSS. ${ }^{*} \mathrm{P}<0.05,{ }^{* * * *} \mathrm{P}<0.001$ vs. 0 min (C) Representative immunoblots and (D) quantitative analysis of PECAM-1 in the HUVECs transfected with NC siRNA or PECAM-1 siRNA after 60-min LSS exposure. ${ }^{*} \mathrm{P}<0.05,{ }^{* * * * *} \mathrm{P}<0.001$ vs. NC+LSS 0 min or as indicated. HUVECs, human umbilical vein endothelial cells; PECAM-1, platelet endothelial cell adhesion molecule-1; LSS, low shear stress; NC, negative control; siRNA, small interfering RNA.

\section{Discussion}

The role of PECAM-1 in LSS-induced EC apoptosis and monocyte adhesion was the main finding of this study. Maintenance of vascular integrity is a major role of the endothelial barrier (17). Macromolecules and cells leak out of the vascular lumen into the interstitial space, inducing inflammation if the endothelial barrier is damaged (13). This induction of inflammation may lead to chronic vascular diseases, such as atherosclerosis, in which barrier function is disrupted and vessel integrity cannot be restored (17). Anti-inflammatory and anticoagulation states, as well as normal blood flow are maintained as a result of the vascular integrity provided by dormant ECs under appropriate physiological conditions. Once a stimuli is received, ECs are activated and undergo apoptosis, which weakensbarrierfunction, resulting in increased permeability and extravasation of inflammatory cells (predominantly leukocytes) and proteins into the inflamed areas (31). PECAM-1 is crucial for stimuli, such as shear stress, which regulate vascular barrier function. Leukocytes, platelets and ECs express PECAM-1 to regulate cell-cell interactions (32). Emerging evidence has revealed that PECAM-1 produces either proatherogenic or antiatherogenic effects, depending on the local hemodynamic environment (19-21). Specifically, PECAM-1 is proatherogenic in the aortic arch where LSS is present, but antiatherogenic in the descending aorta where high shear stress is present $(20,21)$. Under normal conditions, EC barrier properties are supported by PECAM-1 and are influenced by the actions of extracellular messengers, such as cytokines and vascular endothelial growth factor-A, as well as blood flow (32). Under inflammatory conditions, such as atherosclerotic vessels, mediators, including proinflammatory cytokines, which enhance adhesion properties and decrease vascular integrity, have a large impact on PECAM-1 function. Enhanced leukocyte transendothelial migration levels contribute to atherogenesis and atherosclerotic plaque formation (17). High PECAM-1 expression has been observed in vascular cells, including leukocytes, platelets and other blood-borne cells, as well as ECs. Junctional and adhesive properties in ECs are controlled by PECAM-1. Under certain physiological conditions, PECAM-1 also supports the endothelial barrier function (33-35). However, atherosclerosis causes vessel inflammation, which impairs the function of PECAM-1, leading to an increase in leukocytes adhered to ECs, vascular integrity loss and increased transmigration of leukocytes to the intima media (18). The present study demonstrated that LSS enhanced PECAM-1 expression in a time-dependent manner in HUVECs. Knockdown of PECAM-1 inhibited LSS-induced EC apoptosis and monocyte adhesion. 
A
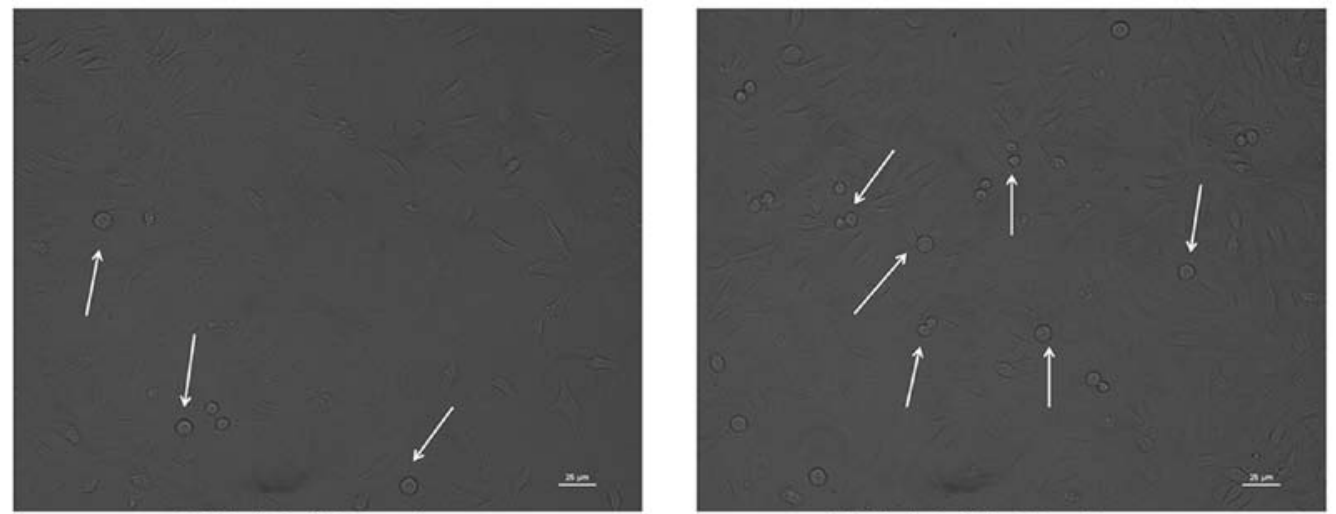

$\mathrm{NC}+\mathrm{LSS} 0 \mathrm{~min}$

$\mathrm{NC}+\mathrm{LSS} 60 \mathrm{~min}$
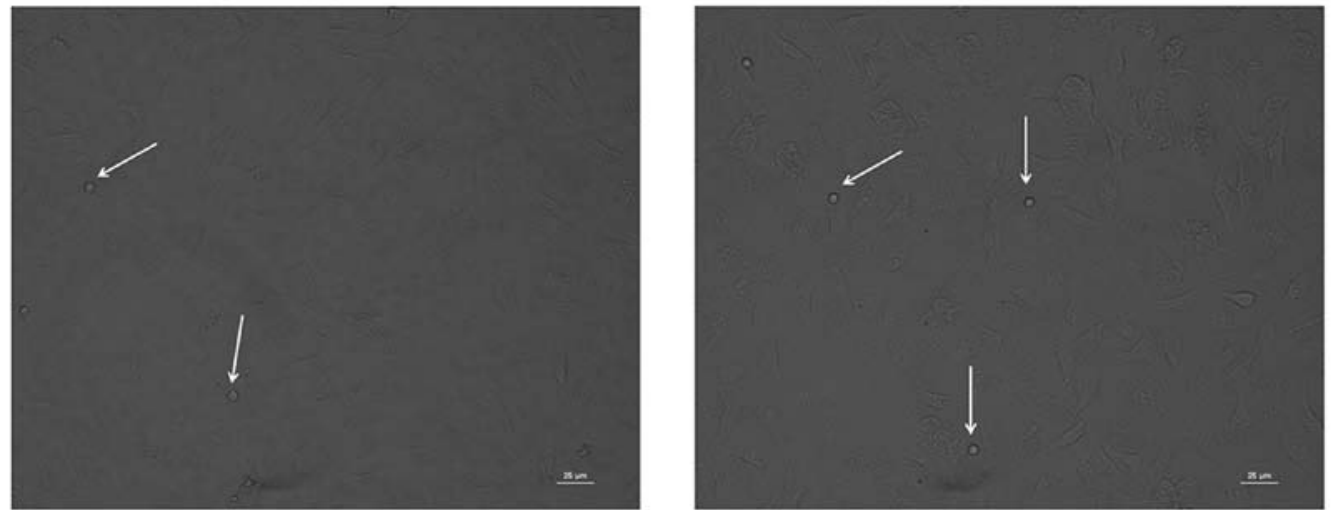

PECAM-1 siRNA + LSS 0min

PECAM-1 siRNA+LSS 60min

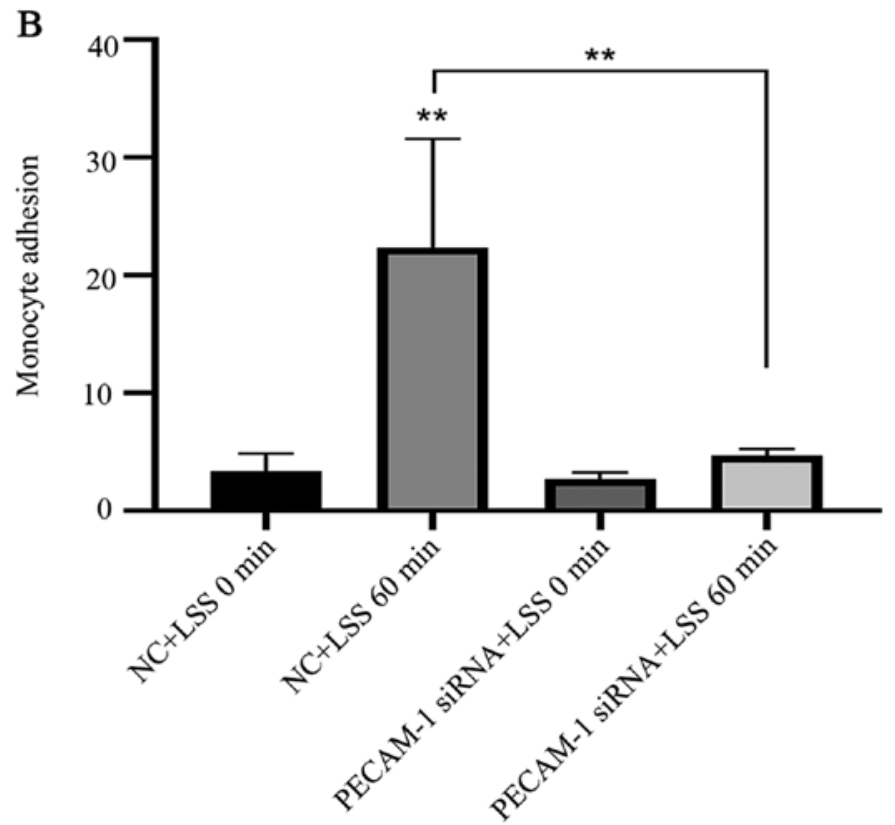

Figure 3. Knockdown of PECAM-1 inhibits LSS-induced adhesion of monocytes to HUVECs. (A) Representative micrographs of monocyte adhesion to the HUVECs. The small round cells are adhered THP-1 monocytic cells (arrowheads). Cells of sample fields at high magnification (x200) are presented. Scale bar, $25 \mu \mathrm{m}$. (B) Total numbers of adherent cells in a total of three randomly selected microscopic fields. ${ }^{* *} \mathrm{P}<0.01$ vs. NC+LSS 0 min or as indicated. HUVECs, human umbilical vein endothelial cells; PECAM-1, platelet endothelial cell adhesion molecule-1; LSS, low shear stress; NC, negative control; siRNA, small interfering RNA.

Previous studies have demonstrated that PECAM-1 modulates the phosphorylation of Akt (17,36,37), which initiates the phosphorylation of FoxO and subsequently rapid relocation to the cytoplasm from the nucleus, leading to the inhibition of pro-apoptotic activity (38). Based on these findings, the present study speculated that the Akt/FoxO1 signaling pathway may be involved in PECAM-1-mediated LSS-induced HUVEC apoptosis. The present study further demonstrated that PECAM-1 had an effect on the phosphorylation of Akt, indicating that PECAM-1 plays a role in the activation of Akt under LSS. As downstream targets of Akt (serine/threonine protein kinase $\mathrm{B}$ or PKB),FoxO transcription factors cause apoptosis and quiescence by limiting cell proliferation (38). The present study also demonstrated that the phosphorylation of FoxO1 occurred 


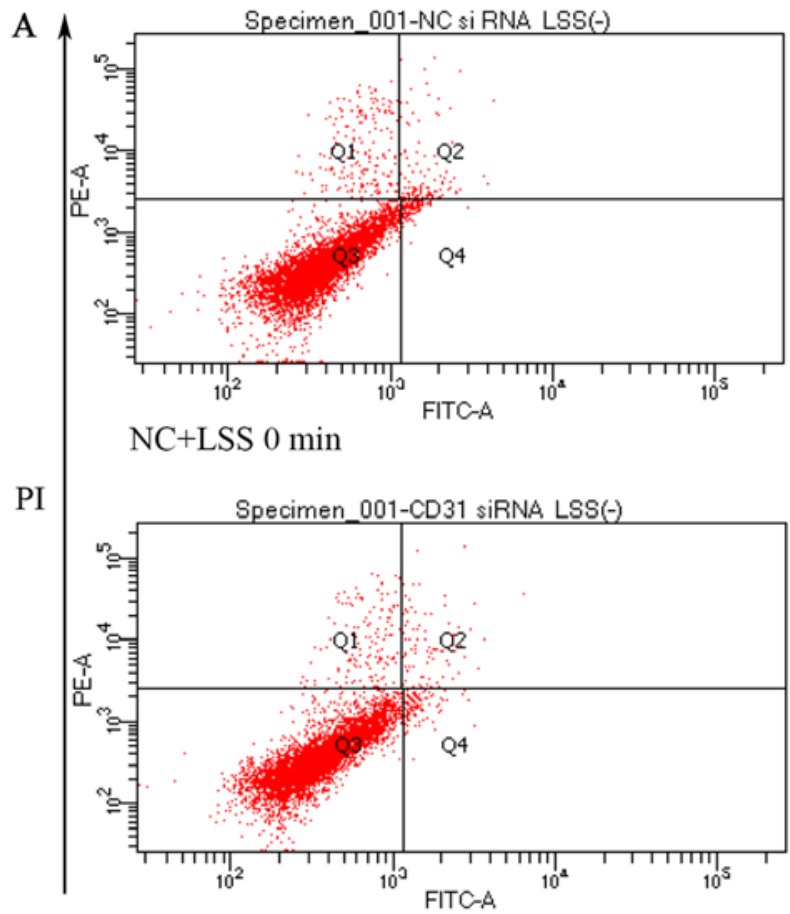

PECAM-1 siRNA+LSS 0 min

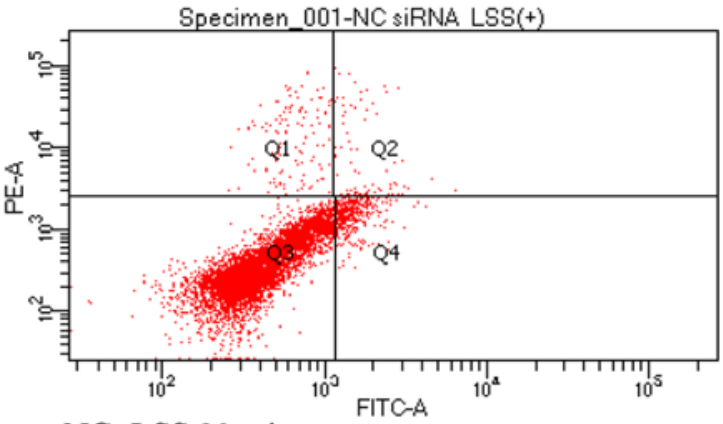

$\mathrm{NC}+\mathrm{LSS} 90 \mathrm{~min}$

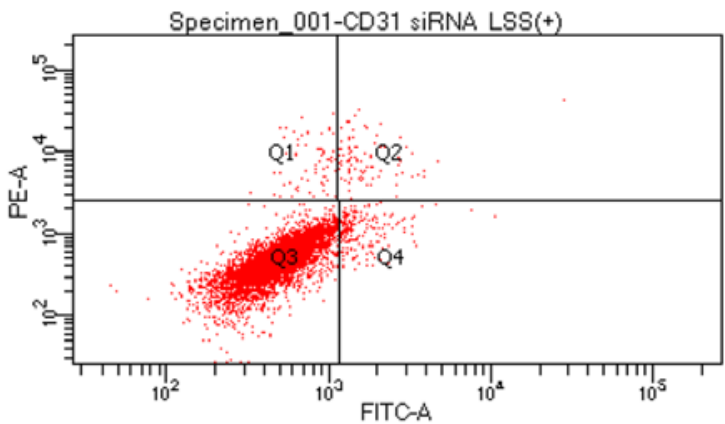

PECAM-1 siRNA+LSS $90 \mathrm{~min}$

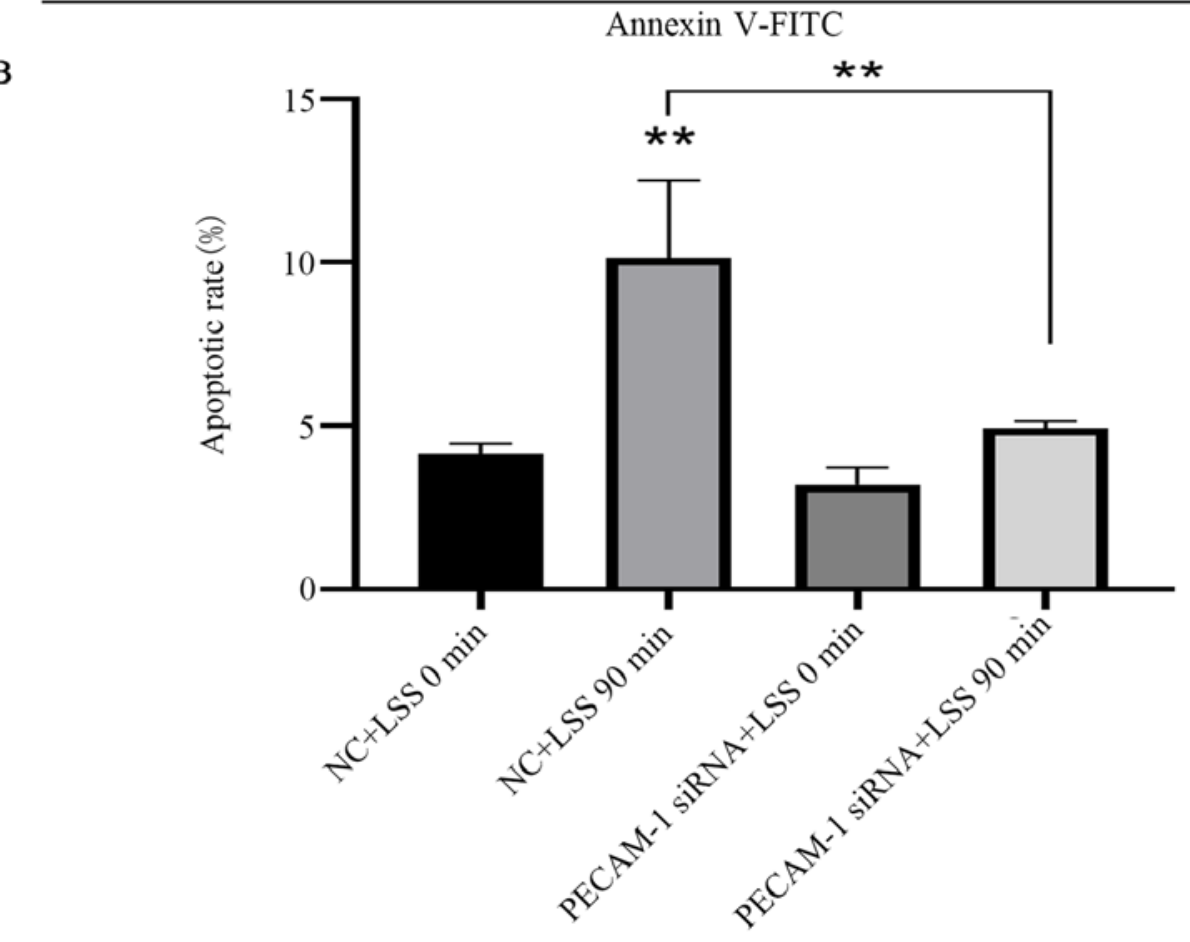

Figure 4. Knockdown of PECAM-1 inhibits LSS-induced apoptosis of HUVECs. (A) Representative flow cytometry blots of HUVECs transfected with NC or PECAM-1 siRNA after 90 min of LSS exposure. (B) Quantification of the apoptosis data. ${ }^{* *} \mathrm{P}<0.01 \mathrm{vs}$. NC+LSS 0 min or as indicated. HUVECs, human umbilical vein endothelial cells; PECAM-1, platelet endothelial cell adhesion molecule-1; LSS, low shear stress; NC, negative control; siRNA, small interfering RNA; PI, propidium iodide.

in the same direction as Akt phosphorylation. Phosphorylation of FoxOs by Akt leads to nuclear exclusion and inactivation, inhibiting FoxO transcriptional functions, while enhancing cell proliferation, growth and survival (38-40). It was further confirmed that FoxO1 relocalization from the nucleus to the cytoplasm followed the phosphorylation of FoxO1. FoxOs can induce the expression of multiple proapoptotic proteins of the mitochondria-targeting $\mathrm{Bcl} 2$ family, whereas the expression of death receptor ligands are stimulated to enhance apoptosis signaling and/or cell growth inhibition (38). A method by which cell survival is promoted by Akt is the sequestration of FoxOs from apoptotic gene promoters. Therefore, FoxOs can drive the expression of multiple apoptotic genes by functioning as important Akt signaling effector arms (41-43). Previous studies have suggested that FoxO1 is phosphorylated and translocated to the cytoplasm from the nucleus, causing FoxO1-mediated 
A

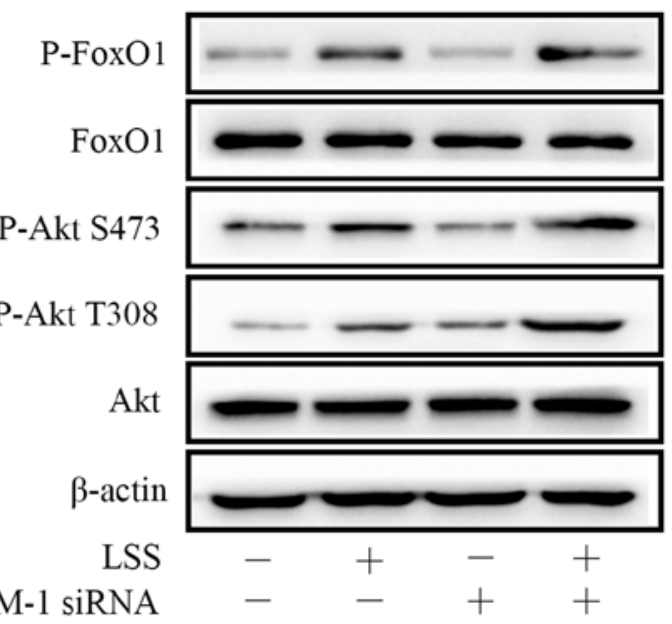

C

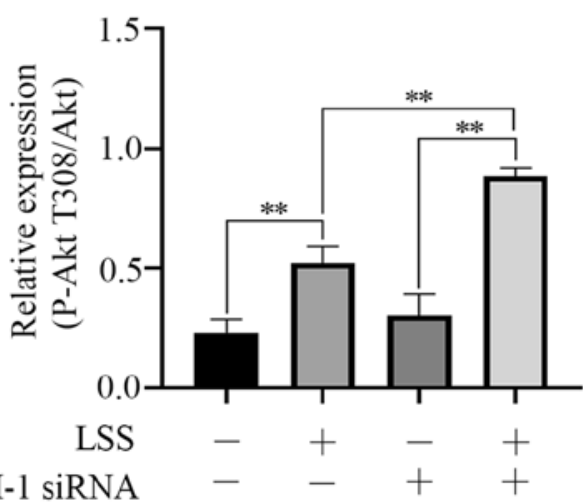

D

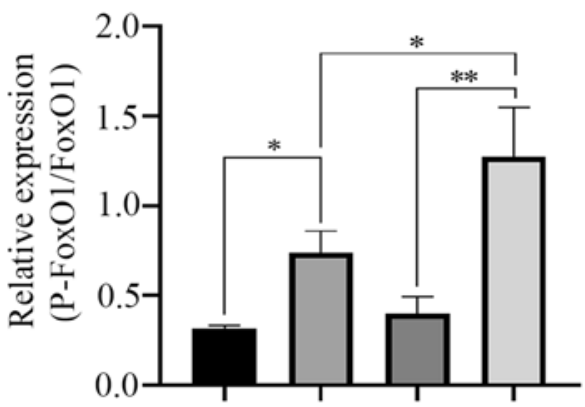

LSS
B

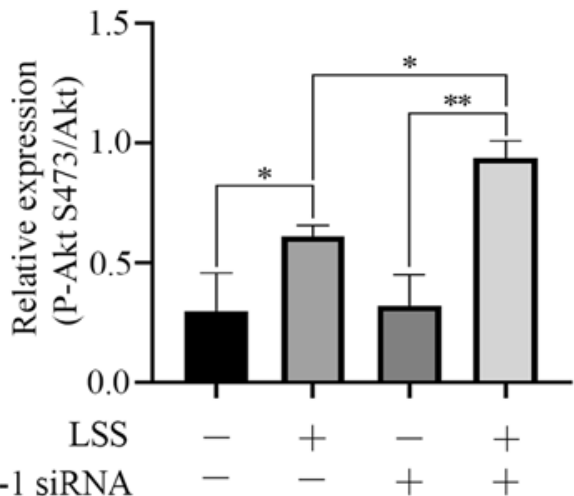

PECAM-1 SiRNA $\quad-\quad-\quad+\quad+$

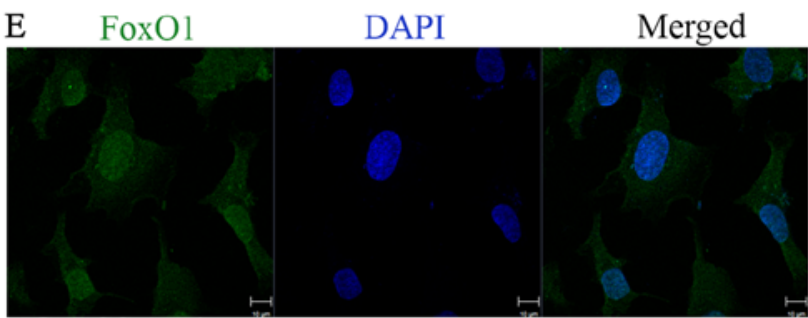

$\mathrm{NC}+\mathrm{LSS} 0 \mathrm{~min}$

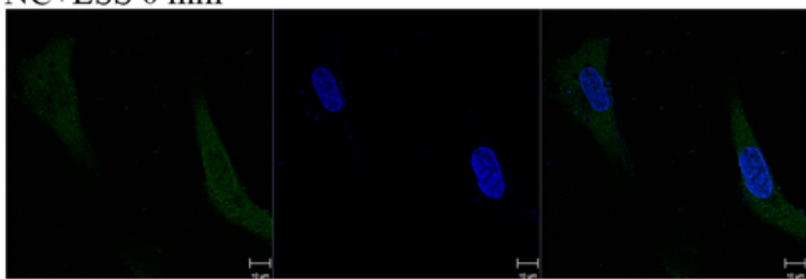

$\mathrm{NC}+\mathrm{LSS} 60 \mathrm{~min}$

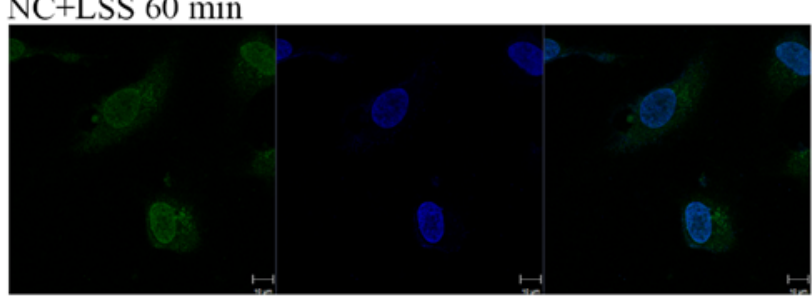

PECAM-1 siRNA+LSS 0 min

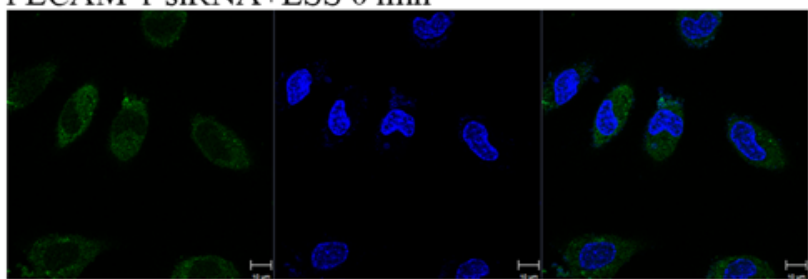

PECAM-1 siRNA+LSS $60 \mathrm{~min}$

Figure 5. PECAM-1 knockdown enhances LSS-induced Akt and FoxO1 phosphorylation in HUVECs. (A) Representative immunoblots and (B-D) quantitative analysis of (B) phospho-Akt Ser473, (C) phospho-Akt Thr308 and (D) phospho-FoxO1 in HUVECs transfected with NC or PECAM-1 siRNA after 60 min of LSS exposure. (E) Immunofluorescence assay was to determine the nuclear translocation of FoxO1 (green). Nuclei were stained with DAPI (blue) Original magnification, x630; scale bar, $10 \mu \mathrm{m} .{ }^{*} \mathrm{P}<0.05,{ }^{* *} \mathrm{P}<0.01$. HUVECs, human umbilical vein endothelial cells; PECAM-1, platelet endothelial cell adhesion molecule-1; LSS, low shear stress; P-, phospho-; FoxO1, forkhead box O1.

apoptosis inhibition $(42,44)$. The results of the present study demonstrated that PECAM-1 knockdown further promoted the phosphorylation of Akt/FoxO1 and mitigated LSS-induced apoptosis of HUVECs, which suggested that PECAM-1 knockdown inhibiting LSS-induced HUVEC apoptosis may be relevant to the Akt/FoxO1 signaling pathway.
In conclusion, the present study revealed that PECAM-1 promoted LSS-induced EC apoptosis and monocyte adhesion. PECAM-1 knockdown inhibiting LSS-induced apoptosis of ECs may be mediated by enhancing the Akt/FoxO1 signaling pathway activation. The results also indicated that PECAM-1 may be a promising target for the treatment of atherosclerosis. 


\section{Acknowledgements}

The authors would like to thank Dr Jian Li (Department of Immunology) and Dr Yuelin Chao (Department of Cardiology, Nanjing First Hospital, Nanjing Medical University, Nanjing, China) for their technical assistance.

\section{Funding}

This study was supported by the National Natural Science Foundation of China (grant nos. 81700239 and 81770441).

\section{Availability of data and materials}

The datasets used during the present study are available from the corresponding author on reasonable request.

\section{Authors' contributions}

XX, FW and SC designed the study. XX, FW, LZ, HY, DP, YL, $\mathrm{XQ}, \mathrm{YG}$ and XL performed the experiments. XX performed the data analysis. XX and SC drafted the manuscript. All authors read and approved the final manuscript.

\section{Ethics approval and consent to participate}

The present study was approved by the Institutional Animal Care and Use Committee of Nanjing Medical University (approval no. SYXK2016-0006).

\section{Patient consent for publication}

Not applicable.

\section{Competing interests}

The authors declare that they have no competing interests.

\section{References}

1. Malekmohammad K, Sewell RDE and Rafieian-Kopaei M: Antioxidants and atherosclerosis: Mechanistic aspects. Biomolecules 9: E301, 2019.

2. Farmakis TM, Soulis JV, Giannoglou GD, Zioupos GJ and Louridas GE: Wall shear stress gradient topography in the normal left coronary arterial tree: Possible implications for atherogenesis. Curr Med Res Opin 20: 587-596, 2004.

3. Sandoo A and Kitas GD: A methodological approach to non-invasive assessments of vascular function and morphology. J Vis Exp 2015.

4. Gimbrone MA Jr and Garcia-Cardena G: Endothelial cell dysfunction and the pathobiology of atherosclerosis. Circ Res 118: 620-636, 2016.

5. Huynh DTN and Heo KS: Therapeutic targets for endothelial dysfunction in vascular diseases. Arch Pharm Res 42: 848-861, 2019.

6. Heo KS, Berk BC and Abe J: Disturbed flow-induced endothelial proatherogenic signaling via regulating post-translational modifications and epigenetic events. Antioxid Redox Signal 25: 435-450, 2016.

7. Penna C, Tullio F, Femmino S, Rocca C, Angelone T, Cerra MC, Gallo MP, Gesmundo I, Fanciulli A, Brizzi MF, et al: Obestatin regulates cardiovascular function and promotes cardioprotection through the nitric oxide pathway. J Cell Mol Med 21: 3670-3678, 2017.

8. Godo S and Shimokawa H: Divergent roles of endothelial nitric oxide synthases system in maintaining cardiovascular homeostasis. Free Radic Biol Med 109: 4-10, 2017.
9. Forstermann $\mathrm{U}, \mathrm{Xia} \mathrm{N}$ and $\mathrm{Li} \mathrm{H}$ : Roles of vascular oxidative stress and nitric oxide in the pathogenesis of atherosclerosis. Circ Res 120: 713-735, 2017.

10. Kumar A, Hung OY, Piccinelli M, Eshtehardi P, Corban MT, Sternheim D, Yang B,Lefieux A,Molony DS, Thompson EW, et al: Low coronary wall shear stress is associated with severe endothelial dysfunction in patients with nonobstructive coronary artery disease. JACC Cardiovasc Interv 11: 2072-2080, 2018.

11. Siasos G, Sara JD, Zaromytidou M, Park KH, Coskun AU, Lerman LO, Oikonomou E, Maynard CC, Fotiadis D, Stefanou K, et al: Local low shearstress and endothelial dysfunction in patients with nonobstructive coronary atherosclerosis. J Am Coll Cardiol 71: 2092-2102, 2018.

12. Plank MJ, Wall DJ and David T: Atherosclerosis and calcium signalling in endothelial cells. Prog Biophys Mol Biol 91: 287-313, 2006

13. Oakley R and Tharakan B: Vascular hyperpermeability and aging. Aging Dis 5: 114-125, 2014.

14. Green J, Yurdagul A Jr, McInnis MC, Albert P and Orr AW: Flow patterns regulate hyperglycemia-induced subendothelial matrix remodeling during earlyatherogenesis. Atherosclerosis 232: 277-284, 2014

15. Chao Y, Zhu L, Qu X, Zhang J, Zhang J, Kong X, Gu Y, Pu J, $\mathrm{Wu} \mathrm{W}$, Ye $\mathrm{P}$, et al: Inhibition of angiotension II type 1 receptor reduced human endothelial inflammation induced by low shear stress. Exp Cell Res 360: 94-104, 2017.

16. Feng YM, Chen XH and Zhang X: Roles of PECAM-1 in cell function and disease progression. Eur Rev Med Pharmacol Sci 20: 4082-4088,2016.

17. Chistiakov DA, Orekhov AN and Bobryshev YV: Endothelial PECAM-1 and its function in vascular physiology and atherogenic pathology. Exp Mol Pathol 100: 409-415, 2016.

18. Lertkiatmongkol P, Liao D, Mei H, Hu Y and Newman PJ: Endothelial functions of platelet/endothelial cell adhesion molecule-1 (CD31). Curr Opin Hematol 23: 253-259, 2016.

19. Harry BL, Sanders JM, Feaver RE, Lansey M, Deem TL, Zarbock A, Bruce AC, Pryor AW, Gelfand BD, Blackman BR, et al: Endothelial cell PECAM-1 promotes atherosclerotic lesions in areas of disturbed flow in ApoE-deficient mice. Arterioscler Thromb Vasc Biol 28: 2003-2008, 2008.

20. Goel R, Schrank BR, Arora S, Boylan B, Fleming B, Miura H, Newman PJ, Molthen RC and Newman DK: Site-specific effects of PECAM-1 on atherosclerosis in LDL receptor-deficient mice. Arterioscler Thromb Vasc Biol 28: 1996-2002, 2008.

21. Harrison M, Smith E, Ross E, Krams R, Segers D, Buckley CD, Nash GB and Rainger GE: The role of platelet-endothelial cell adhesion molecule- 1 in atheroma formation varies depending on the site-specific hemodynamic environment. Arterioscler Thromb Vasc Biol 33: 694-701, 2013.

22. Zhang J, Wang Z, Zhang J, Zuo G, Li B, Mao W and Chen S: Rapamycin attenuates endothelial apoptosis induced by low shear stress via mTOR and sestrin1 related redox regulation. Mediators Inflamm 2014: 769608, 2014.

23. Rezvan A, Ni CW, Alberts-Grill $\mathrm{N}$ and Jo $\mathrm{H}$ : Animal, in vitro, and ex vivo models of flow-dependent atherosclerosis: Role of oxidative stress. Antioxid Redox Signal 15: 1433-1448, 2011

24. Ramkhelawon B, Vilar J, Rivas D, Mees B, de Crom R, Tedgui A and Lehoux S: Shear stress regulates angiotensin type 1 receptor expression in endothelial cells. Circ Res 105: 869-875, 2009.

25. Avivi C, Rosen O and Goldstein RS: New chromogens for alkaline phosphatase histochemistry: Salmon and magenta phosphate are useful for single- and double-label immunohistochemistry. J Histochem Cytochem 42: 551-554, 1994.

26. Specht E, Kaemmerer D, Sanger J, Wirtz RM, Schulz S and Lupp A: Comparison of immunoreactive score, HER2/neu-Score and $\mathrm{H}$-Score for the immunohistochemical evaluation of somatostatin receptors in bronchopulmonary neuroendocrine neoplasms. Histopathology 67: 368-377, 2015.

27. Zhang M, Sun J, Chen B, Zhao Y, Gong H, You Y and Qi R: Ginkgolide B inhibits platelet and monocyte adhesion in TNF $\alpha$-treated HUVECs under laminar shear stress. BMC Complement Altern Med 18: 220, 2018.

28. Davis FM and Gallagher KA: Epigenetic mechanisms in monocytes/macrophages regulate inflammation in cardiometabolic and vascular disease. Arterioscler Thromb Vasc Biol 39: 623-634, 2019.

29. Fong GH: Potential contributions of intimal and plaque hypoxia to atherosclerosis. Curr Atheroscler Rep 17: 510, 2015.

30. Dong G, Yang S, Cao X, Yu N, Yu J and Qu X: Low shear stress-induced autophagy alleviates cell apoptosis in HUVECs. Mol Med Rep 15: 3076-3082, 2017. 
31. Danese S, Dejana E and Fiocchi C: Immune regulation by microvascular endothelial cells: Directing innate and adaptive immunity, coagulation, and inflammation. J Immunol 178: 6017-6022, 2007.

32. Sarelius IH and Glading AJ: Control of vascular permeability by adhesion molecules. Tissue Barriers 3: e985954, 2015.

33. Muller WA, Weigl SA, Deng X and Phillips DM: PECAM-1 is required for transendothelial migration of leukocytes. J Exp Med 178: 449-460, 1993.

34. Privratsky JR, Paddock CM, Florey O, Newman DK, Muller WA and Newman PJ: Relative contribution of PECAM-1 adhesion and signaling to the maintenance of vascular integrity. J Cell Sci 124: 1477-1485, 2011.

35. Privratsky JR and Newman PJ: PECAM-1: Regulator of endothelial junctional integrity. Cell Tissue Res 355: 607-619, 2014.

36. Fleming I, Fisslthaler B, Dixit M and Busse R: Role of PECAM-1 in the shear-stress-induced activation of Akt and the endothelial nitric oxide synthase (eNOS) in endothelial cells. J Cell Sci 118: 4103-4111, 2005

37. Jones CI, Sage T, Moraes LA, Vaiyapuri S, Hussain U, Tucker KL, Barrett NE and Gibbins JM: Platelet endothelial cell adhesion molecule-1 inhibits platelet response to thrombin and von Willebrand factor by regulating the internalization of glycoprotein Ib via AKT/glycogen synthase kinase-3/dynamin and integrin aIIb33. Arterioscler Thromb Vasc Biol 34: 1968-1976, 2014.

38. Zhang X, Tang N, Hadden TJ and Rishi AK: Akt, FoxO and regulation of apoptosis. Biochim Biophys Acta 1813: 1978-1986, 2011.
39. Wilhelm K, Happel K, Eelen G, Schoors S, Oellerich MF, Lim R, Zimmermann B, Aspalter IM, Franco CA and Boettger T, et al: FoxO1 couples metabolic activity and grow th state in the vascular endothelium. Nature 529: 216-220, 2016.

40. Dharaneeswaran H, Abid MR, Yuan L, Dupuis D, Beeler D, Spokes KC, Janes L, Sciuto T, Kang PM, Jaminet SS, et al: FoxO1-mediated activation of Akt plays a critical role in vascular homeostasis. Circ Res 115: 238-251,2014.

41. Li F, Qu H, Cao HC, Li MH, Chen C, Chen XF, Yu B, Yu L, Zheng LM and Zhang W: Both FoxO3a and FoxO1 are involved in the HGF-protective pathway against apoptosis in endothelial cells. Cell Biol Int 39: 1131-1137, 2015.

42. Xing YQ, Li A, Yang Y, Li XX, Zhang LN and Guo HC: The regulation of FoxO1 and its role in disease progression. Life Sci 193: 124-131,2018.

43. Fu Z and Tindall DJ: FoxOs, cancer and regulation of apoptosis. Oncogene 27: 2312-2319, 2008.

44. Cifarelli V, Lee S, Kim DH, Zhang T, Kamagate A, Slusher S, Bertera S, Luppi P, Trucco M and Dong HH: FoxO1 mediates the autocrine effect of endothelin-1 on endothelial cell survival. Mol Endocrinol 26: 1213-1224, 2012.

(i)(9) This work is licensed under a Creative Commons EY NG NO Attribution-NonCommercial-NoDerivatives 4.0 International (CC BY-NC-ND 4.0) License. 